\title{
Energy levels of Neutrinos in a Gravitational Potential Well
}

\author{
Leon F. Phillips ${ }^{1}$ \\ ${ }^{1}$ University of Canterbury, Christchurch, New Zealand \\ Correspondence: Leon F. Phillips, University of Canterbury, Christchurch, New Zealand. E-mail: \\ leon.phillips@canterbury.ac.nz
}

Received: December 6, 2014 Accepted: December 18, 2014 Online Published: December 23, 2014

doi:10.5539/apr.v7n1p19

URL: http://dx.doi.org/10.5539/apr.v7n1p19

\begin{abstract}
Arguments are presented against the existence of mathematical singularities in the physical universe. Energy levels are calculated for neutrinos in the gravitational potential wells of black holes of sizes ranging from one hundred to one hundred million solar masses, with up to $\sim 10^{60}$ energy levels available below the event horizon and with the capacity to hold two identical fermion neutrinos, with opposite spin vectors, in each energy level. Because of the very high abundance of neutrinos in the universe, it is suggested that the gravitational wells of the larger black holes are likely to be full of neutrinos up to and beyond their event horizons. This might provide a means of extracting information from inside the event horizon of a large black hole with an advanced neutrino telescope.
\end{abstract}

Keywords: Mathematical singularities and the Heisenberg uncertainty principle, Black Hole cores, gravitational potential wells, neutrinos and the Pauli principle, information extraction from a Black Hole

\subsection{Introduction}

Outstanding problems in connection with black holes and gravitation include the mathematical singularity that classical gravitation theory predicts to exist at the center of a black hole, the inability of an observer outside the event horizon of a black hole to discover what is happening inside, the mysterious information loss from behind an event horizon when a black hole dissolves into Hawking radiation, the nature of the dark matter that is a major constituent of the universe, and the form of the interface between quantum mechanics and Einstein's theory of gravitation. Clearly not all of these problems can be solved at once. The purpose of this note is to point out that clues showing the way to plausible solutions exist for several of these problems, and neutrinos are involved in most of them.

A first clue is provided by the conclusion (Dolgov, 2008) that neutrinos, with an abundance of several hundred per cubic centimeter, are the second most numerous particles in the universe, the most numerous being photons of the background microwave radiation. A second clue is the experimental observation (Bilenky, 2011) that neutrinos have a non-zero rest mass, which permits them to travel at a speed that is much less than the speed of light. Like everything else, neutrinos can be captured by black holes, for which process they would seem to be ideal test particles. At present not much can be made of the observation that neutrinos have sufficient internal structure to allow them to exist in at least three different flavors (Halzen, 2013), but no doubt time will tell. A more helpful clue is provided by the well-known text-book calculation of effective potentials for light test particles in the vicinity of a black hole (Misner, Thorne, \& Wheeler, 1973, p. 659), and another by the fact that most, or possibly all, neutrinos are fermions (Dolgov, 2008), which can occupy a range of energy levels in the effective potentials, each level holding at most two neutrinos with opposite spins in accordance with the Pauli exclusion principle. Here we consider only fermion neutrinos, and we do not discuss the complications that could arise from the possibility of transitions between different flavors (Medvedev, 2012). One more clue is provided by the circumstance that the effective potentials are approximately of the form $V(r)=A / r+O\left(1 / r^{2}\right)$, where $A$ is a known constant and the calculation of energy levels for this kind of system is another well-known text-book example.

\subsection{On Singularities}

The basis of the present argument against the existence of mathematical singularities in the physical universe is the assertion that the small-scale properties of the universe are entirely determined by quantum mechanics. Therefore in the event of a disagreement between quantum mechanics and a classical theory such as general relativity, concerning a small-scale phenomenon like a singularity, quantum mechanics must prevail.

The singularities at $r=0$ in black hole solutions of Einstein's general relativity equations would be a serious problem if the solutions did actually apply at $r=0$, because they would contradict the extremely well-tested 
Heisenberg uncertainty principle, which states that the product of the statistical uncertainties in the values of a pair of measurable quantities, such as position and momentum, or energy and time, whose quantum-mechanical operators do not commute, must be greater than a definite small multiple of Planck's constant. Therefore a mathematical singularity in one member of a pair, in this case position, necessarily implies an infinite value for the variability of the other, namely momentum. That is not acceptable, because there is not enough space-time inside the event horizon of a black hole, nor even in a finite physical universe, to accommodate an infinitely variable momentum. This argument holds unless the universe is actually infinite, which does not seem to be the case, or unless pairs of measurable quantities like position and momentum, or mass-energy and time, lose their identity inside a black hole, for which there is no evidence. Hence a mathematical derivation that leads to a conclusion which violates the Heisenberg principle must be based on one or more incorrect assumptions.

In addition to this theoretical prohibition of mathematical singularities in the physical universe, astronomical observations (R. Schödel et al., 2002) have shown for the black hole Saggitarius A*, located near the center of our galaxy, that a major fraction of the mass-energy of the presumed product of gravitational collapse remains invisible behind the event horizon after the collapse, and can be detected by its effect on the motion of nearby stars. Therefore it has not obeyed the mathematics by disappearing into a singularity, which implies that there is no singularity to disappear into.

According to some accounts, the passage of an object through a black hole event horizon, on the way to being crushed to nothing in the central singularity, occurs infinitely slowly from the viewpoint of an external observer, which is equivalent to saying that it never occurs within the lifetime of the external universe in which we live. This author takes the simpler position that there are no mathematical singularities in the physical universe because that would violate the Heisenberg principle.

It therefore follows that the residue of a massive, gravitationally-collapsed object must consist of a spheroidal lump or core of ultra-dense matter. The physics and chemistry of the ultra-dense matter are at present unknown but are not necessarily unknowable and should be very interesting. The various space-time metrics with a singularity at $r=0$ do apply in a near-vacuum, but evidently they do not apply inside a lump of ultra-dense matter, and we shall assume that their range of application commences at the value of $r$ that corresponds to the surface of the spheroidal core.

\section{Model Calculations}

We begin with the effective potential $V(r)$ for a neutrino in the region outside the event horizon of a Schwartzschild non-rotating black hole. This is given implicitly by

$$
V(r)^{2}=(1-2 M / r)\left(m^{2}+L^{2} / r^{2}\right)
$$

where $m$ is the reduced mass of the neutrino-black hole system, which is effectively the mass of the neutrino, $M$ is the mass of the black hole core, and $L$ is the angular momentum of the neutrino as it approaches the black hole (Misner, Thorne, \& Wheeler, 1973). Note that $V(r)$ is expressed in the 'geometric' units of metres, where 1 metre $=1.210 \times 10^{41}$ kilojoules, and $M / r$ is dimensionless. For a particle crossing the event horizon where $r=2 M$ in the Schwartzschild metric, the $r$ coordinate changes its nature from spacelike to timelike, and Equation (1) passes through a coordinate singularity that can be eliminated by transforming to Eddington-Finkelstein coordinates, with a new time coordinate, or to Kruskal-Szekeres coordinates, with a $z-w$ plane in place of the $r-t$ plane of the Schwartzschild system. The present model calculations do not distinguish between time and space coordinates, the symbol $r$ being employed merely for convenience, and we could equally well use $s$ or $\tau$. Equation (1) continues to hold inside the Schwartzschild event horizon, but the physical natures of $V(r)$ and $r$ change.

We calculate the total mass-energy $V(r)$ of the neutrino approaching the black hole as the square root of the absolute value of the right-hand side of Equation (1), for $r$ values that can be regarded as a parameterization of proper time $\tau$ values for a neutrino that is gravitating towards the core. Eventually a fermion neutrino has to stop because there are no unoccupied energy levels available at lower mass-energy. The mass-energy of the neutrino then has a quite well-defined value given by $V(r)$, and the time coordinate has a corresponding large Heisenberg uncertainty. By this process, a quasi-equilibrium distribution of neutrinos over the mass-energy levels in the potential well can develop over some astronomical period of time.

Figures. 1a-d show plots of the effective potential $V(r)$ given by Equation (1) versus $1 / r$ for a neutrino inside the event horizons of some representative black holes. These plots are not perfectly linear, but the deviation from linearity is probably small enough to be regarded as a perturbation. For Figs. $1 \mathrm{~b}$ to $1 \mathrm{~d}$ the curvature would make the energy levels crowd together slightly at higher energy. 


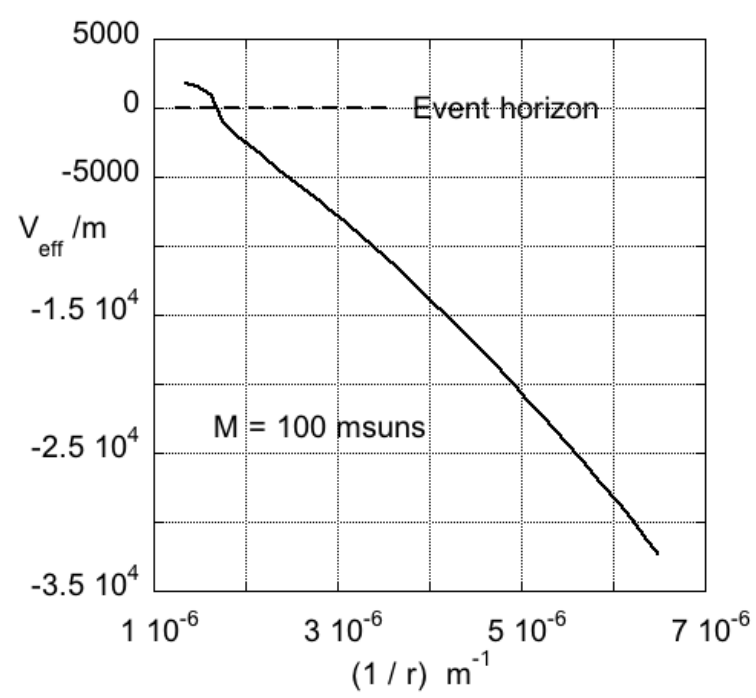

Figure 1a

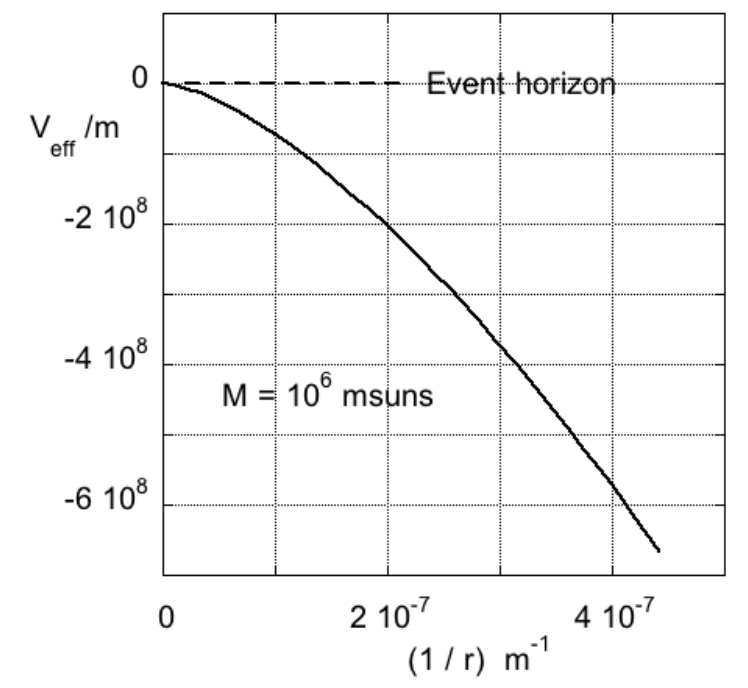

Figure 1c

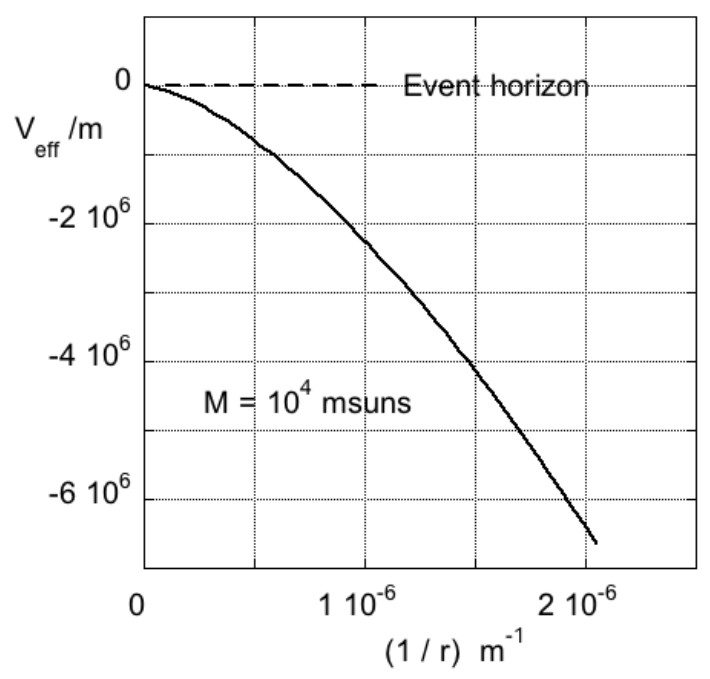

Figure 1b

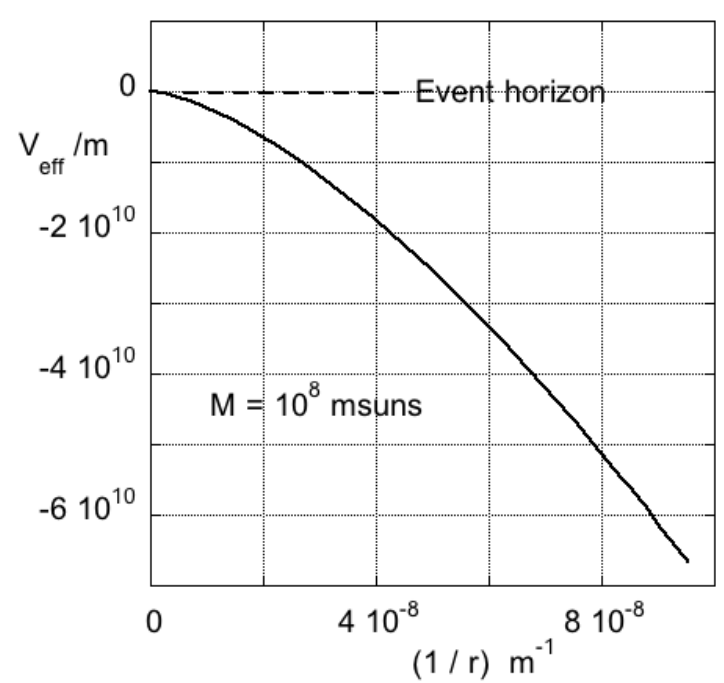

Figure 1d

Figures 1 a to $1 \mathrm{~d}$, plots of Veff versus $1 / r$ for black hole core masses of $10^{2}, 10^{4}, 10^{6}$ and $10^{8}$ times the solar mass. Note that the effective potential is expressed in the 'geometrical' units of metres, where 1 metre $=1.210 \times 10^{41}$ kilojoules

An important difference between the systems black-hole-plus-neutrinos and positive-nucleus-plus-electrons is that neutrinos carry no electric charge, so they repel one another only via the short-range Fermi hole that does not permit the close approach of a pair of identical fermion neutrinos unless they have opposite spins. Hence the wave function for a neutrino in a multi-neutrino system with spherical symmetry is almost independent of the number of neutrinos in the system, and consists essentially of a spherical harmonic $Y_{l m}(\theta, \phi)$ multiplied by a radial function based on an associated Laguerre polynomial in the variable $r$, together with a normalizing factor $N_{n l m}$ where $n$ is the principal quantum number. The resulting energy levels of a neutrino near a black hole are analogous to the electronic energy levels of a hydrogen atom, which are given by

$$
E_{n}=-h^{2} / 8 \pi^{2} \mu a_{0}^{2} n^{2}
$$

Here $\mu$, the reduced mass of the system electron + nucleus, is practically the same as the mass of the electron, $a_{0}$ is the Bohr radius $h^{2} / 4 \pi^{2} \mu \varepsilon^{2}$, where $\varepsilon$ is the charge on the electron, and the principal quantum number $n$ ranges from 1 to infinity. The energy levels for a given value of $n$ are degenerate, apart from small effects such as Lamb shifts, the number of available levels at a particular value of $n$ being $n^{2}$, and each level can hold at most two identical fermions with opposite spins.

Equation (2) results from application of the Schrödinger equation to the potential energy given by 


$$
V(r)=-\varepsilon^{2} / r
$$

The equation corresponding to (3) for the black hole-plus-neutrino system is approximately

$$
V(r)=-G M m / r
$$

where $G$ is the gravitational constant, $M$ the mass of the black hole and $m$ the rest mass of the neutrino. Hence the Bohr radius for this system is $h^{2} / 4 \pi^{2} G M m^{2}$ and the equation corresponding to (2) is

$$
E_{n}=-2 \pi^{2} G^{2} M^{2} m^{3} / h^{2} n^{2}
$$

The energy levels given by Equation (5) are incorrect for a relativistic system because they converge to zero as $n$ goes to infinity, whereas a proper relativistic treatment would show them converging to the rest mass-energy of the test particle, as in the following expression for the relativistic energy levels of a hydrogen-like atom

$$
E_{n}=m c^{2}\left[1-(Z \alpha)^{2} / 2 n^{2}-\ldots \text { fine-structure terms }\right]
$$

where $\alpha^{2} m c^{2} / 2=\varepsilon^{2} / 8 \pi^{2} a_{0}$ and $Z$ is the charge on the nucleus (Sakurai, 1967). However, the mass-energy of a slow neutrino, ca. $0.2 \mathrm{eV}$ (King and Luhn, 2013), which can be compared with $0.5 \mathrm{MeV}$ for an electron, is so small in comparison with the energies associated with the potential well of Equation (4) that for our present purpose, which is to obtain qualitative insight rather than precise numbers, the approximation of ignoring the rest-mass is tolerable. The convergence of $E_{n}$ towards the neutrino's rest mass occurs for energy levels that are above the event horizon of the black hole, so the number of available fermion states in the potential well at levels up to and including the event horizon must be large but finite. Above the event horizon the levels merge into a quasi-continuum in which the neutrinos are only weakly bound to the core. An incidental consequence of the neutrino's tiny mass is that the possibility that the arrival of slow neutrinos will excite quasi-normal modes of vibration of the black hole (Cho, 2003) can generally be ignored.

The density assumed for the ultra-dense matter of which the black hole core is composed has an effect on the results of model calculations because it controls the core radius, which is the minimum radius at which Equation (1) applies. A typical density for a neutron star (Trümper, 2011) is about $3 \times 10^{15} \mathrm{~kg} / \mathrm{m}^{3}$, but that is unlikely to represent the upper limit for a black hole core. The present calculations arbitrarily assume that the density increases in proportion to the mass of the core to the power 0.15 , beginning at a density of about $10^{16} \mathrm{~kg} / \mathrm{m}^{3}$ for a core mass equal to thirty solar masses. A more likely scenario would have the density increase discontinuously with increasing mass of the core, in steps of unknown size, in accordance with an unknown ultra-high-pressure phase diagram (Weber, 2005). Fortunately the results of the calculations are not very sensitive to the core radius.

A question that arises naturally with this simple model is that the effective potential of Equation (1) was intended for describing the motion of macroscopic test particles in the gravitational field of a body such as the sun, whereas a neutrino weighs much less than an electron, so surely its motion should be described by the Dirac equation? The short answer is that the Dirac theory of fermions is not a single-particle theory, but rather deals with a multi-component Dirac field that operates on state vectors in occupation-number space through the agency of creation and annihilation operators (Sakurai, 1967). This formalism is entirely compatible with the present model, which considers the occupation by neutrinos of the available energy levels inside the gravitational potential well of a black hole.

A more serious problem is that Equation (1) is probably not an accurate description of the world inside the event horizon. To quote J.J. Sakurai (1967), "In relativistic field theory we visualize the interactions as arising from the exchange of quanta." Thus the concept of a potential plays little or no part in most calculations for relativistic fields. Nevertheless the topology of the black hole plus neutrino system dictates that there must be a mass-energy $V(r), V(s)$ or $V(\tau)$ that varies with the proper time $\tau$ associated with the trajectory of an incoming neutrino, and $V(\tau)$ must resemble the $1 / r$ potential that is used as an approximation to Equation (1), in that it decreases monotonically between the event horizon and the black hole core, and can be approximated by a near-linear plot of mass-energy versus $1 / r$. That is all we ask of it at present.

Another problem with the present model is that we are assuming that the distribution of neutrinos over energy levels is essentially static, despite the likelihood that the neutrinos in the lowest levels will always be in the process of being incorporated into the core of the black hole. For a small, newly-formed black hole the rate of assimilation of neutrinos from the background population, which is about 300 particles per cubic centimeter (Dolgov, 2008), would probably not be sufficient to maintain a high proportion of filled energy levels inside the event horizon. However, as the results in Table 1 show, the ratio of the area of the event horizon to the surface area of the solid core increases very rapidly with black-hole mass, so that a quasi-equilibrium distribution of neutrinos over energy levels inside the event horizon almost certainly exists for a super-massive black hole such as Saggitarius $A^{*}\left(\sim 10^{6}\right.$ solar masses). 
Table 1. Results of model calculations. $\left[1.30(16)=1.30 \times 10^{16}\right]$

\begin{tabular}{ccclcccc}
\hline$m_{\text {core }} / m_{\text {sun }}$ & $\rho_{\text {core }} / \mathrm{kg} \mathrm{m}^{-3}$ & $r_{\text {core }} / m$ & $L_{\text {om }} / \mathrm{m}^{2} \mathrm{~s}^{-1}$ & $V_{\text {well }} / \mathrm{kJ}$ & $N_{\text {levels }}$ & $f_{\text {well }}$ & $A_{\text {ratio }}$ \\
\hline $1.0(2)$ & $1.20(16)$ & $1.58(5)$ & $8.86(7)$ & $1.09(44)$ & $1.20(65)$ & 0.027 & $3.48(0)$ \\
$1.0(3)$ & $1.69(16)$ & $3.04(5)$ & $8.86(8)$ & $1.50(45)$ & $6.15(63)$ & 0.0081 & $9.45(1)$ \\
$1.0(4)$ & $2.39(16)$ & $5.84(5)$ & $8.86(9)$ & $1.83(46)$ & $2.61(62)$ & 0.0051 & $2.56(3)$ \\
$1.0(5)$ & $3.38(16)$ & $1.12(6)$ & $8.86(10)$ & $2.19(47)$ & $1.08(61)$ & 0.0043 & $6.95(4)$ \\
$1.0(6)$ & $4.77(16)$ & $2.15(6)$ & $8.86(11)$ & $2.60(48)$ & $4.43(59)$ & 0.0041 & $1.89(6)$ \\
$1.0(7)$ & $6.74(16)$ & $4.13(6)$ & $8.86(12)$ & $3.09(49)$ & $1.81(58)$ & 0.0041 & $5.11(7)$ \\
$1.0(8)$ & $9.52(16)$ & $7.93(6)$ & $8.86(13)$ & $3.68(50)$ & $7.53(56)$ & 0.0041 & $1.39(9)$ \\
\hline
\end{tabular}

Table 1 contains results of model calculations of energy levels for neutrinos near Schwartzschild black holes having masses $m_{\text {core }}$ that range from $10^{2}$ to $10^{8}$ solar masses. Calculations which vary $L_{o m}$, the product of the impact parameter $b_{\text {value }}$ and the neutrino velocity $v_{\text {particle }}$, over a large range lead to the conclusion that the effect of the neutrino's angular momentum on the potential is very small for a massive black hole. In the table, $V_{\text {well }}$ is the depth of the gravitational potential well; $r_{\text {core }}$ is the radius of the solid core of the black hole; $N_{\text {levels }}$ is the number of energy levels below the event horizon, the sums of the degeneracies $n^{2}$ being calculated by the finite-difference method (for comparison, the number of 'background' neutrinos inside the volume of the event horizon of the largest black hole in the table is of the order of $\left.10^{23}\right) ; f_{\text {well }}$ is the fraction of the potential well that is above the event horizon; $A_{\text {ratio }}$ is the area of the spherical event horizon divided by the surface area of the spheroidal core. The depths of the potential wells and the numbers of energy levels that they contain below the event horizon are very large, but not unreasonably so in view of the astronomical time and distance scales that are involved.

A large $A_{\text {ratio }}$ value implies that neutrinos can be gathered into the event horizon at a much faster rate than they can be absorbed into the core, so that a quasi-equilibrium distribution can develop. The increase in $A_{\text {ratio }}$ with increasing $m_{\text {core }}$ is so dramatic that one cannot escape the conclusion that large black holes must inevitably become filled with neutrinos to a level above the event horizon. The prediction that the number of energy levels inside the well actually decreases with increasing mass of the black hole core is an unanticipated consequence of the increase in the spacing of energy levels with increasing mass. However, this prediction is quite sensitive to the assumed dependence of core density on mass, which used the arbitrary formula

$$
\rho_{\text {core }}=10^{16}\left(m_{\text {core }} / 30\right)^{0.15}
$$

Whether or not the prediction is correct, it is clear that the number of available levels does not increase rapidly with the core mass, which implies that a large and growing black hole should emit a steady flow of neutrinos. A listing of the computer program with instructions for running these calculations is available on request from the author.

Analogous calculations could be made for a Kerr black hole. One of the interesting characteristics of an extreme Kerr black hole is the phenomenon of frame-dragging, such that a test particle or an observer finds itself in a local frame that is spinning very rapidly relative to the external universe. From the point of view of an observer within the spinning frame it would appear to be the universe that was spinning so that, at least as a first guess, such an observer might expect Equation (1) and deductions from it to continue to be valid for quantities measured relative to the local frame.

\section{Conclusions}

The existence of a mathematical singularity at the center of a black hole is ruled out by the Heisenberg uncertainty principle and, albeit less decisively, by the astronomical observations of the Saggitarius A* black hole. The nature of the interface between quantum mechanics and classical gravitation theory evidently is such that a classical theory like General Relativity cannot be expected to work properly on the scale at which the Heisenberg principle is important. Dyson (2013) has shown that a quantum theory of gravitation based on gravitons is probably not viable because gravitons are almost certainly undetectable, but a definite conclusion depends on work that is still in progress. The present paper might be regarded as representing a small extension of the domain of quantum mechanics into the field of gravitation and cosmology. The problem of how an outside observer can obtain information from inside a black hole and the related information-loss problem are solved in principle if we assume that the older black holes in the universe have had time to capture sufficient neutrinos to fill their energy levels to a point above the event horizon, so that someone from an advanced civilization might use a neutrino telescope (Margiotta et al., 2014) to interrogate the neutrinos in the upper levels for information about what is happening in 
the interior, the Pauli principle providing the necessary link between energy levels inside and outside the event horizon. This implies, in effect, that 'hair' on a black hole exists and is composed of neutrinos, and that a black hole that is in the process of disappearing by emission of Hawking radiation is liable to be a very hairy specimen indeed. Because the number of available energy levels below the event horizon either decreases or at most increases only slowly with increasing core mass (depending on the method of calculating $\rho_{\text {core }}$ ), an actively growing massive black hole should emit a steady flux of neutrinos. Regarding the problem of dark matter, the smallness of the neutrino rest mass would appear to rule out clouds of slow neutrinos as candidates for the invisible glue that holds galaxies together. Thus one might argue that the neutrino family needs to be extended to include bosonic or fermionic WIMPs (Gould, 1992). However, other conclusions are possible (Parkhomov, 2010), and the present situation is quite fluid.

\section{Acknowledgment}

This work was supported by the University of Canterbury. The author is grateful for informative discussions with Professor David Wiltshire.

\section{References}

Halzen, F. (2013). The IceCube Collaboration. Journal of Physics: Conference Series, 408, 012023/1-012023/4.

A. Margiotta, on behalf of the KM3NeT Collaboration (2014). Detection potential of the KM3NeT detector for high-energy neutrinos from the Fermi bubbles. arXiv:1408.1392v1 [astro-ph.IM] 5 Aug 2014.

Bilenky, S. M. (2011). Neutrino masses and oscillations. arXiv:1105.2306v1 [hep-ph] 11 May 2011.

Cho, H. T. (2003). Dirac quasinormal modes in Schwarzschild black hole spacetimes. Physical Review D, 68(2), 024003. http://dx.doi.org/10.1103/PhysRevD.68.024003

Dolgov, A. D. (2008). Cosmology and Neutrino Properties, Physics of Atomic Nuclei, 71, 2152-2164.

Dyson, F. (2013). Is a graviton detectable? International Journal of Modern Physics, 28, 1330041-1330055.

Gould, A. (1992). Cosmological density of WIMPs from solar and terrestrial annihilations. Astrophysics Journal, 388, 338-344. http://dx.doi.org/10.1086/171156

King, S. F., \& Luhn, C. (2013). Neutrino mass and mixing with discrete symmetry. Reports on Progress in Physics, 76(5), 056201. http://dx.doi.org/10.1088/0034-4885/76/5/056201

Medvedev, M. V. (2012). 'Evaporation' of a flavor-mixed particle from a gravitational potential. ArXiv: 1201.5697v1 [astro-ph.CO] 27 Jan 2012.

Misner, C. W., Thorne, K. S., \& Wheeler, J. A., (1973). Gravitation. W.H. Freeman and Company, San Francisco, p. 659 .

Parkhomov, A. G. (2010). Influence of relic neutrinos on beta radioactivity. ArXiv: 1010.1591, 2010.

Sakurai, J. J. (1967). Advanced Quantum Mechanics. Addison-Wesley, Reading Mass..

Schödel, R., Ott, T., Genzel, R., Hofmann, R., Lehnert, M., Eckart, A., ... Menten, K. M. (2002). A star in a 15.2-year orbit around the supermassive black hole at the center of the Milky Way. Nature, 419, 694-696. http://dx.doi.org/10.1038/nature01121

Trümper, J. E. (2011). Observations of neutron stars and the equation of state of matter at high densities, Progress in Particle and Nuclear Physics, 66, 674-680

Weber, F. (2005). Strange quark matter and compact stars. Progress in Particle and Nuclear Physics, 54, 193-288. http://dx.doi.org/10.1016/j.ppnp.2004.07.001

\section{Copyrights}

Copyright for this article is retained by the author(s), with first publication rights granted to the journal.

This is an open-access article distributed under the terms and conditions of the Creative Commons Attribution license (http://creativecommons.org/licenses/by/3.0/). 